\title{
La réforme du système de sécurité sociale
}

\section{Felicia Roșioru}

\section{(2) OpenEdition}

Journals

Édition électronique

URL : https://journals.openedition.org/rdctss/2060

DOI : $10.4000 /$ rdctss. 2060

ISSN : 2262-9815

\section{Éditeur}

Centre de droit comparé du travail et de la sécurité sociale

\section{Édition imprimée}

Date de publication : 1 avril 2018

Pagination : 180-181

ISSN : 2117-4350

\section{Référence électronique}

Felicia Rosioru, «La réforme du système de sécurité sociale », Revue de droit comparé du travail et de la sécurité sociale [En ligne], 1 | 2018, mis en ligne le 01 novembre 2021, consulté le 13 novembre 2021. URL : http://journals.openedition.org/rdctss/2060 ; DOI : https://doi.org/10.4000/rdctss.2060

\section{(c) $(i)(9)$}

Revue de droit comparé du travail et de la sécurité sociale est mise à disposition selon les termes de la Licence Creative Commons Attribution - Pas d'Utilisation Commerciale - Pas de Modification 4.0 International. 


\title{
FELICIA ROSIORU
}

\author{
Université Babes-Bolyal de Cluj-Napoca
}

\section{LA RÉFORME DU SYSTÈME DE SÉCURITÉ SOCIALE}

Les changements législatifs les plus importants au cours du deuxième semestre 2017 concernent le système roumain de la sécurité sociale. À partir du $1^{\text {er }}$ janvier 2018 , les contributions sociales sont passées, à quelques exceptions près, entièrement à la charge du salarié. Celui-ci doit payer $10 \%$ de son revenu pour le système d'assurance-maladie, $25 \%$ pour le fonds des pensions et 10 \% pour l'impôt sur le revenu. Avant 2018, l'employeur et le salarié partageait la charge des contributions sociales. En plus, pour le travail salarié, l'employeur est obligé de payer une contribution de 2,25 \% du fonds mensuel des salaires. Les sommes payées sont divisées entre le fond de garantie des créances salariales (pour couvrir les salaires restant à payer en cas de la faillite de l'employeur) et le système d'assurance contre le chômage, les congés payés en cas de maladie ou maternité et le système d'assurance pour les maladies et les accidents professionnels. Les employeurs qui bénéficient du travail effectué dans des conditions spéciales ou dangereuses (entraînant des risques de travail, ainsi que l'exposition au bruit, à la chaleur, à des substances toxiques etc.) doivent payer une contribution mensuelle supplémentaire de 4 ou $8 \%$, en fonction des conditions du travail. L'obligation de verser cette contribution supplémentaire est fondée sur l'idée que celui qui bénéficie du travail effectué dans des conditions spéciales ou dangereuses doit en supporter la charge financière. Les raisons de cette réforme n'ont jamais été claires, l'idée principale était de faire sortir de l'ombre le travail non-déclaré, dès lors que les employeurs n'ont plus un intérêt fiscal à occulter l'existence ou la nature du contrat de travail.

Cette réforme a été accompagnée par l'augmentation du salaire minimum brut garanti

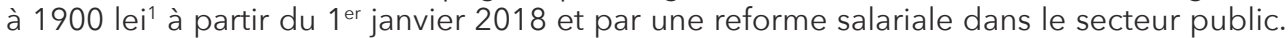
Les salaires ont été - en règle générale - augmentés de manière à couvrir la différence entre les cotisations dues avant le 1er janvier 2018 et à partir de cette date. La déclaration du Gouvernement roumain consistait à apporter une garantie du niveau du salaire reçu le 31 décembre 2017, qui ne devait pas baisser. Pourtant, de nombreux salariés ont vu leurs salaires diminuer et, pour certaines catégories professionnelles, mieux payées la diminution a été importante. Par contre, dans le secteur privé, les salariés n'ont eu aucune garantie contre la baisse de leurs salaires suite à la réforme du système de la sécurité sociale. Normalement, la solution raisonnable aurait dû être la négociation - individuelle et collective. En réalité, parce que la plupart des entreprises sont des petites et moyennes entreprises, souvent il n'existe ni de syndicat ni aucune forme de représentation collective des salariés. Selon la loi roumaine sur le dialogue social, pour former un syndicat il faut au moins 15 salariés travaillant pour un même employeur et les élections pour mettre en place des représentants des salariés sont organisées uniquement à partir de 21 salariés dans l'entreprise.

En plus, à partir du $1^{\mathrm{e}}$ janvier 2018, toutes les personnes physiques résidant en Roumanie sont assurées du système d'assurance-maladie et du fonds des pensions, et sont obligées de payer les contributions correspondantes (10\% et $25 \%$ ) pour tous les revenus mensuels tirés des activités professionnelles supérieurs au salaire minimum garanti (actuellement de 1900 lei). Auparavant, seule l'assurance-maladie était obligatoire pour les personnes ayant des revenus professionnels, indépendamment du type d'activité (salariée ou indépendante). Les personnes ayant le statut d'indépendant avaient le droit

1 Ce qui équivaut environ à 415 euros. 
de conclure des contrats pour bénéficier des pensions, des indemnités de chômage ou d'arrêts de travail indemnisés en cas de maladie, de maternité ou pour soigner un enfant malade, âgé de moins de 7 ans. Certaines catégories de personnes sont exemptées de l'obligation de contribuer au système d'assurance-maladie: les mineurs; les jeunes âgés de moins de 26 ans, s'ils sont élèves, étudiants ou apprentis et n'ont pas de revenus professionnels; les jeunes âgés de moins de 26 ans provenant du système de protection des enfants; les personnes persécutées durant le régime communiste pour des raisons politiques; les personnes handicapées ${ }^{2}$; l'époux, l'épouse et les parents d'un(e) assuré(e); les femmes enceintes; les bénéficiaires du revenu minimum garanti; les détenus et ceux qui sont en détention provisoire; les citoyens roumains victimes du trafic de personnes; le personnel des cultes reconnus par la loi etc. La réforme du système de la sécurité sociale a été accompagnée de la réforme des lois régissant les différentes prestations sociales (loi n 95/2006 sur l'assurance -maladie, la loi $n^{\circ}$ 263/2010 sur le système unitaire des pensions, loi $n^{\circ} 76 / 2002$ sur le système d'assurance-chômage, loi $n^{\circ}$ 346/2002 relative aux assurances en cas de maladies professionnelles et accidents du travail).

Les mesures législatives adoptées ont conduit à supprimer le financement des systèmes d'assurance par des autorités ou institution publiques en cas de maladie, maternité, congé parental ou de chômage de l'assuré. À partir du $1^{\text {er }}$ janvier 2018, les bénéficiaires de l'indemnité maladie, maternité, congé parental ou de l'indemnité chômage restent des assurés du système public de la sécurité sociale, mais la contribution correspondante (10\% pour les assurances maladie et $25 \%$ pour le fond des pensions) est déduite de l'indemnité perçue. Auparavant, les bénéficiaires des indemnités susmentionnées préservaient leur qualité d'assurés du système de sécurité sociale, mais la contribution correspondante était assumée par l'autorité en charge d'administrer la prestation sociale octroyée (par exemple, la Caisse d'assurance-maladie). En plus, la loi contenait une disposition discriminatoire visà-vis des salariés à temps partiel. lls étaient obligés de payer des taxes et des cotisations sociales mensuelles correspondant au salaire minimum garanti ${ }^{3}$, indépendamment du nombre d'heures de travail effectuées par jour ou par semaine. Suite à la réforme du 8 février 20184, les cotisations des travailleurs à temps partiel sont calculées sur le salaire minimum garanti, mais sont dues par l'employeur. Ceci tend, d'une part, à privilégier les salariés à temps partiel par rapport aux autres salariés qui supportent entièrement la charge de leurs assurances sociales. D'autre part, ce changement législatif a provoqué le licenciement massif de salariés à temps partiel en raison du coût pour l'employeur et est à l'origine de la fermeture de nombreux petits restaurants ainsi qu'à la cessation d'autres activités connaissant un fort taux de travail à temps partiel.

La réforme du système de la sécurité sociale a été très contestée parce qu'elle a été mal expliquée, presque non-motivée et elle a abouti, dans certains cas, à la diminution des droits sociaux de certaines catégories professionnelles. En plus, la réforme n'a été assortie d'aucune garantie pour les salariés du secteur privé contre la baisse de leur salaire. Les politiques menées par le Gouvernement roumain sont assez fluctuantes; la réforme de la sécurité sociale comprend elle-même un certain degré d'imprévisibilité.

2 Les personnes handicapées ne sont exemptées de l'obligation de contribuer au système d'assurance-maladie que pour certains revenus (les allocations octroyées par les autorités publiques), mais elles doivent contribuer au système d'assurance-maladie pour les revenus professionnels.

3 Les impôts et les cotisations sociales mensuelles représentent 45 \% du salaire mensuel (soit par rapport au salaire minimum garanti, 855 lei; ce qui équivaut à environ 185 euros).

4 Ordonnance d'urgence du Gouvernement n³/2018. 